\title{
ARCHIMEDES INTEGRALS AND CONUCLEAR SPACES
}

\author{
B. JEFFERIES and S. OKADA \\ (Received 30 April 1987; revised 30 March 1988) \\ Communicated by R. O. Vyborny
}

\begin{abstract}
The lack of completeness with respect to the semivariation norm, of the space of Banach space valued functions, Pettis integrable with respect to a measure $\mu$, often impedes the direct extension of results involving integral representations, true in the finite-dimensional setting, to the general vector space setting. It is shown here that the space of functions with values in a space $Y$, $\mu$-Archimedes integrable in a Banach space $X$ embedded in $Y$, is complete with respect to convergence in semivariation, provided the embedding from $X$ into $Y$ is completely summing. The result is applied to the case when $Y$ is a conuclear space, in particular, when $X$ is a function space continuously included in a space of distributions.
\end{abstract}

1980 Mathematics subject classification (Amer. Math. Soc.) (1985 Revision): primary 38 B 05 , 46 G 10; secondary 47 B 10, 46 A 12.

Keywords and phrases: Archimedes integral, completeness, convergence in mean, absolutely summing, conuclear space.

\section{Introduction}

It is well known that the basic difference between the Pettis and Bochner integrals of functions with values in an infinite dimensional Banach space $X$ is that the vector space of equivalence classes of Pettis integrable functions is not complete in the topology of convergence in semivariation, yet the space of Bochner integrable functions is complete with respect to the $L^{1}$-norm. The completeness of the space of integrable functions is used extensively in applications of integration theory.

The first author was supported by a Queen Elizabeth II Research Fellowship.

(C) 1989 Australian Mathematical Society 0263-6115/89 \$A2.00+0.00 
The Archimedes integral was introduced in [4] to compensate for this deficiency of the Pettis integral. The Archimedes integral has values in the Banach space $X$, but the integrand is allowed to take its values in a larger space $Y$ in which $X$ is embedded. For the appropriate choice of the locally convex space $Y$, and up to sets of measure zero, the space $\mathfrak{L}(\lambda ; X, Y)$ of functions Archimedes integrable with respect to a measure $\lambda$ is complete.

Our concern in this note is with the choice of the space $Y$. It is shown in [4] that there always exists a space $Y$ into which $X$ embeds, for which $\mathfrak{L}(\lambda ; X, Y)$ is complete, but $Y$ may be too large; for example, the product space $\mathbb{C}^{\Gamma}$ with $\Gamma$ a separating family of continuous linear functionals on $X$. In Theorem 6 , we show that in the case when $Y$ is a Banach space, if the embedding from $X$ into $Y$ is absolutely summing, then $\mathfrak{L}(\lambda ; X, Y)$ is complete. The conclusion holds under somewhat weaker conditions, which are explored in Section 3. A natural choice for $Y$ is a space of distributions for which $X$ is continuously included in $Y$. Section 2 deals with the definition, and basic properties of the Archimedes integral introduced in [4].

Some terminology adopted throughout this paper follows.

Let $\Omega$ be a non-empty set. For ease of notation, subsets of $\Omega$ will be identified with their characteristic functions. Let $\mathfrak{A}$ be a $\sigma$-algebra of subsets of $\Omega$.

Given a Banach space $X$ with norm $|\cdot|$ and dual space $X^{\prime}$, the variation $|m|$ of a vector measure $m: \mathfrak{A} \rightarrow X$ is defined to be the smallest non-negative measure which dominates the set function $E \rightarrow|m(E)|, E \in \mathfrak{A}$. For each $x^{\prime} \in X^{\prime}$, let $\left\langle m, x^{\prime}\right\rangle$ be the complex-valued measure defined by $\left\langle m, x^{\prime}\right\rangle(E)=$ $\left\langle m(E), x^{\prime}\right\rangle, E \in \mathfrak{A}$. The semivariation $\|m\|$ of $m$ is defined by

$$
\|m\|(E)=\sup \left\{\left|\left\langle m, x^{\prime}\right\rangle\right|(E):\left|x^{\prime}\right| \leq 1, x^{\prime} \in X^{\prime}\right\}, \quad E \in \mathfrak{A} .
$$

\section{The Archimedes integral}

Let $\lambda: \mathfrak{A} \rightarrow[0, \infty]$ be a measure. The following lemma is well known.

Lemma 1. Suppose that $c_{j} \in X$ are vectors and $E_{i} \in \mathfrak{A}$ are sets, $i=1,2, \ldots$, such that

$$
\sum_{i \in N}\left|c_{i}\right| \lambda\left(E_{i}\right)<\infty
$$

and the equality $\sum_{i \in \mathbf{N}} c_{i} E_{i}(\omega)=0$ holds for every $\omega \in \Omega$ satisfying the relation

$$
\sum_{i \in N}\left|c_{i}\right| E_{i}(\omega)<\infty
$$

Then the equality $\sum_{i \in N} c_{i} \lambda\left(E_{i}\right)=0$ holds. 
Recall that a strongly $\lambda$-measurable function $f$ on $\Omega$ is said to be Bochner $\lambda$-integrable if the function $|f|$ is $\lambda$-integrable [2, Chapter 11].

According to [3], a function $f: \Omega \rightarrow X$ is Bochner $\lambda$-integrable if and only if there exist vectors $c_{i} \in X$ and sets $E_{i} \in \mathfrak{A}, i=1,2, \ldots$, such that (1) holds and the equality $f(\omega)=\sum_{i \in N} c_{i} E_{i}(\omega)$ holds for every $\omega \in \Omega$ satisfying (2). For such a function $f$, the indefinite Bochner integral $f \lambda: \mathfrak{A} \rightarrow X$ is defined by

$$
f \lambda(E)=\sum_{i \in \mathbb{N}} c_{i} \lambda\left(E_{i} \cap E\right), \quad E \in \mathfrak{A} .
$$

The set function $f \lambda$ is well defined by Lemma 1 , and it is $\sigma$-additive by the Vitali-Hahn-Saks Theorem [2, Corollary 1.5.10]. The space of $X$-valued Bochner $\lambda$-integrable functions on $\Omega$ is denoted by $\mathfrak{B}(\lambda, X)$. Define the Bochner seminorm $|\cdot|_{1}$ on the space $\mathfrak{B}(\lambda, X)$ by $|f|_{1}=|f \lambda|(\Omega)$, for each $f \in \mathfrak{B}(\lambda, X)$.

The next lemma is proved similarly to Theorem 3 of [4]; it is the Beppo Levi theorem for Bochner integrable functions.

LeMMA 2. Suppose that $f_{n}: \Omega \rightarrow X, n=1,2, \ldots$, are Bochner $\lambda$-integrable functions for which $\sum_{n \in N}\left|f_{n}\right|_{1}<\infty$. Let $f: \Omega \rightarrow X$ be a function such that

$$
f(\omega)=\sum_{n \in N} f_{n}(\omega)
$$

for every $\omega \in \Omega$ for which $\sum_{n \in \mathrm{N}}\left|f_{n}(\omega)\right|<\infty$. Then the function $f$ is Bochner $\lambda$-integrable and

$$
\lim _{N \rightarrow \infty}\left|f-\sum_{n=1}^{N} f_{n}\right|_{1}=0 .
$$

Let $Y$ be a locally convex space for which there exists a continuous embed$\operatorname{ding} J: X \rightarrow Y$. A function $f: \Omega \rightarrow Y$ is called Archimedes integrable with respect to $\lambda$ in the space $X$, briefly, $(J, X, \lambda)$-integrable, if there exist vectors $c_{i} \in X$ and sets $E_{i} \in \mathfrak{A}, i=1,2, \ldots$, satisfying the following conditions:

(A1) the sequence $\left\langle c_{i} \lambda\left(E_{i}\right)\right\rangle_{i \in N}$ is unconditionally summable in the space $X$, and

(A2) if $y^{\prime} \in Y^{\prime}$, then

$$
\left\langle f(\omega), y^{\prime}\right\rangle=\sum_{i \in N}\left\langle J\left(c_{i}\right), y^{\prime}\right\rangle E_{i}(\omega)
$$

for every $\omega \in \Omega$ for which $\sum_{i \in N}\left|\left\langle J\left(c_{i}\right), y^{\prime}\right\rangle\right| E_{i}(\omega)<\infty$.

The set valued function $f \lambda: \mathfrak{A} \rightarrow X$ defined by

$$
f \lambda(E)=\sum_{i \in \mathbb{N}} c_{i} \lambda\left(E \cap E_{i}\right), \quad E \in \mathfrak{A},
$$


is called the indefinite Archimedes integral of the function $f$ with respect to $\lambda$. By Lemma 1 , the indefinite integral $f \lambda$ is a well defined set function, because $Y^{\prime}$ separates points of $J(X)$. By the Vitali-Hahn-Saks Theorem, it is $\sigma$-additive.

The vector space of all $Y$-valued $(J, X, \lambda)$-integrable functions on $\Omega$ is denoted by $\mathfrak{L}(\lambda ; J, X, Y)$. Define the seminorm $\|\cdot\|$ on $\mathfrak{L}(\lambda ; J, X, Y)$ by

$$
\|f\|=\|f \lambda\|(\Omega), \quad f \in \mathfrak{L}(\lambda ; J, X, Y) .
$$

The topology on $\mathfrak{L}(\lambda ; J, X, Y)$ given by this seminorm is called the topology of convergence in mean. Whenever there is no danger of confusion, the reference to the embedding $J$ is omitted.

If we let $\mathfrak{A}_{\lambda}=\{E \in \mathfrak{A}: \lambda(E)<\infty\}$, then it is clear that the image of the space $\operatorname{sim}\left(\mathfrak{A}_{\lambda}, X\right)$ of $X$-valued $\mathfrak{A}_{\lambda}$-simple functions on $\Omega$, via the embedding $J$, is dense in the space $\mathfrak{L}(\lambda ; J, X, Y)$ [4, Proposition 2].

If a function $f: \Omega \rightarrow X$ is Bochner $\lambda$-integrable, then it belongs to $\mathfrak{L}(\lambda ; X, X)$ and its indefinite Bochner integral is identical with its indefinite Archimedes integral.

There always exists a locally convex space $Y$, and a continuous embedding $J$ of $X$ into $Y$ such that the space $\mathfrak{L}(\lambda ; J, X, Y)$ is complete with respect to convergence in the mean. For example, if $\Gamma$ is a subset of $X^{\prime}$ separating points of $X$, then define $J_{\Gamma}: X \rightarrow \mathbb{C}^{\Gamma}$ by

$$
J_{\Gamma}(x)(\xi)=\langle x, \xi\rangle
$$

for every $x \in X$ and $\xi \in \Gamma$. The map $J_{\Gamma}$ is a continuous embedding of $X$ into the product space $\mathbb{C}^{\Gamma}$, and we have the following

Theorem 3 [4, Theorem 5]. Let $X$ be a Banach space and let $\Gamma$ be a subset of $X^{\prime}$ which separates points of $X$. Then the space $\mathfrak{L}\left(\lambda ; J_{\Gamma}, X, \mathbb{C}^{\Gamma}\right)$ is complete with respect to the topology of convergence in mean for every non-negative measure $\lambda$.

\section{Completely summing operators}

Let $Y$ be a sequentially complete locally convex Hausdorff space. The linear subspace of $Y$ spanned by a closed, bounded and disked (that is, balanced and convex) subset $B$ of $Y$ is denoted by $Y_{B}$. The space $Y_{B}$, equipped with the gauge $|\cdot|_{B}$ of $B$, is a Banach space [1, Lemma III.3.1].

Let $X$ be a Banach space. Let $\mathfrak{S}$ be the family of all closed, bounded and disked subsets of $Y$. A continuous linear map $T$ from $X$ into $Y$ is called 
completely summing if for each unconditionally summable sequence $\left\langle x_{n}\right\rangle_{n \in N}$ in $X$, there exists a set $B$ in $\mathfrak{S}$ such that the sequence $\left\langle T x_{n}\right\rangle_{n \in \mathbb{N}}$ lies in $Y_{B}$ and $\sum_{n \in N}\left|T x_{n}\right|_{B}<\infty$.

The set $B$ above may depend on the sequence $\left\langle x_{n}\right\rangle_{n \in N}$ in $X$. If there exists a single set $B$ in $\mathfrak{S}$ such that for each unconditionally summable sequence $\left\langle x_{n}\right\rangle_{n \in N}$ in $X$, the sequence $\left\langle T x_{n}\right\rangle_{n \in N}$ lies in $Y_{B}$ and $\sum_{n \in N}\left|T x_{n}\right|_{B}<\infty$, then the map $T$ is said to be totally summing (see [8, page 69]).

A sequence $\left\langle x_{n}\right\rangle_{n \in N}$ in the locally convex space $Y$ is said to be absolutely summable, if for every continuous seminorm $p$ on $Y, \sum_{n \in \mathrm{N}} p\left(x_{n}\right)<\infty$. Recall that a continuous linear map from $X$ into $Y$ is said to be absolutely summing if it maps each unconditionally summable sequence to an absolutely summable sequence (see [2, Proposition VI.3.2]). If $Y$ happens to be a Banach space, then a map between $X$ and $Y$ is absolutely summing if and only if it is totally summing. The same holds true for any locally convex space $Y$ with the property $(B)$ introduced by Pietsch $[6,15.5]$, such as a metric or dual metric space.

The following example shows that it can happen that a map is completely summing without itself being totally summing.

EXAMPLE 4. Let $\Upsilon$ be an uncountable index set, and set $X=l^{2}(\Upsilon)$, $Y=\mathbb{C}^{\Upsilon}$. The collection of all closed, bounded and disked subsets of $Y$ is denoted by $\mathfrak{S}$. Let $\left\langle e_{j}\right\rangle_{j \in \Upsilon}$ be the standard orthonormal basis of $l^{2}(\Upsilon)$. The map $J: X \rightarrow Y$ defined by $J(x)=\left\langle x, e_{j}\right\rangle_{j \in \Upsilon}$ for every $x \in X$ is absolutely summing.

Let $\left\langle x_{n}\right)_{n \in N}$ be an unconditionally summable sequence in $X$. Then there exists a countable subset $\Gamma$ of $\Upsilon$ such that $\left\langle x_{n}, e_{j}\right\rangle=0$ for every $j \in \Upsilon \backslash \Gamma$, and every $n \in \mathbf{N}$.

Let $\pi$ be the projection of $\mathbb{C}^{\Upsilon}$ onto $C^{\Gamma}$. Then $\left\langle\pi \circ J\left(x_{n}\right)\right\rangle_{n \in N}$ is an absolutely summable sequence in $C^{\Gamma}$. The space $C^{\Gamma}$ is a Fréchet space, so it has property (B) mentioned earlier [6]. Consequently, there exist a closed, bounded disked subset $B$ of $\mathbb{C}^{\Gamma}$ such that the sequence $\left\langle\pi \circ J\left(x_{n}\right)\right\rangle_{n \in N}$ lies in $\left(\mathbb{C}^{\Gamma}\right)_{B}$ and

$$
\sum_{n \in N}\left|\pi \circ J\left(x_{n}\right)\right|_{B}<\infty
$$

Let $A=B \times\{0\}$ in $\mathbb{C}^{\Upsilon}$. Then $A$ is also closed, bounded and disked, the sequence $\left\langle J\left(x_{n}\right)\right\rangle_{n \in \mathrm{N}}$ lies in $Y_{A}$ and

$$
\sum_{n \in \mathbb{N}}\left|J\left(x_{n}\right)\right|_{A}=\sum_{n \in N}\left|\pi \circ J\left(x_{n}\right)\right|_{B}<\infty .
$$

Thus, $J$ is completely summing. 
On the other hand, $J$ is not totally summing. If so, there would exist a set $B \in \mathfrak{S}$ such that $J: X \rightarrow Y_{B}$ is absolutely summing. Since $X$ is non-separable and reflexive, there is no such map $J$ [5, Corollary 7].

Proposition 5. Let $Y$ be a sequentially complete locally convex Hausdorff space and let $X$ be a Banach space for which there exists a continuous embedding $J$ from $X$ into $Y$. Let $\mathfrak{S}$ be the family of all closed, bounded, disked subsets of $Y$.

Then the following statements are equivalent.

(i) Given a non-negative measure $\lambda$ and a subset of $\Gamma$ of $X^{\prime}$ which separates points of $X$, to each function $f \in \mathfrak{L}\left(\lambda ; J_{\Gamma}, X, \mathbb{C}^{\Gamma}\right)$ there corresponds a set $B \in \mathfrak{S}$ and a function $g \in \mathfrak{B}\left(\lambda, Y_{B}\right)$ such that

$$
J \circ(f \lambda)=g \lambda .
$$

(ii) Let $\lambda$ be the Lebesgue measure on $[0,1]$. To each function $f \in \mathfrak{L}(\lambda ; X, X)$ there corresponds a set $B \in \mathfrak{S}$ and a function $g \in \mathfrak{B}\left(\lambda, Y_{B}\right)$ for which (4) holds.

(iii) The injection $J$ is completely summing.

Proof. It is clear that statement (i) implies statement (ii).

To see that statement (ii) implies (iii), take an unconditionally summable sequence $\left\langle x_{n}\right\rangle_{n \in N}$ from $X$. Let $E_{n}, n=1,2, \ldots$, be pairwise disjoint sets of positive measure, and define the function $f:[0,1] \rightarrow X$ by $f(\omega)=$ $\sum_{n \in \mathrm{N}} x_{n} E_{n}(\omega) / \lambda\left(E_{n}\right), \omega \in[0,1]$. Then $f \in \mathfrak{L}(\lambda ; X, X)$, and so by virtue of (ii), there exists a set $B \in \mathfrak{S}$ and a function $g \in \mathfrak{B}\left(\lambda, Y_{B}\right)$ such that (4) holds. Consequently,

$$
\sum_{n \in N}\left|J\left(x_{n}\right)\right|_{B}=\int_{0}^{1}|g(\omega)|_{B} d \omega<\infty .
$$

Assume now that (iii) holds and let $f \in \mathfrak{L}\left(\lambda ; J_{\Gamma}, X, \mathrm{C}^{\Gamma}\right)$ be a function. Choose vectors $c_{i} \in X$ and sets $E_{i} \in \mathfrak{A}, i=1,2, \ldots$, such that the sequence $\left\langle c_{i} \lambda\left(E_{i}\right)\right\rangle_{i \in N}$ is unconditionally summable in $X$, and for each $\gamma \in \Gamma$, the equality $\langle f(\omega), \gamma\rangle=\sum_{i \in N}\left\langle c_{i}, \gamma\right\rangle E_{i}(\omega)$ holds for every $\omega \in \Omega$ for which $\sum_{i \in \mathrm{N}}\left|\left\langle c_{i}, \gamma\right\rangle\right| E_{i}(\omega)<\infty$.

Since the map $J$ is completely summing, there exists a set $B \in \mathfrak{S}$ such that the sequence $\left\langle J\left(c_{i}\right) \lambda\left(E_{i}\right)\right\rangle_{i \in N}$ lies in $Y_{B}$ and $\sum_{i \in N}\left|J\left(c_{i}\right) \lambda\left(E_{i}\right)\right|_{B}<\infty$.

Define the function $g: \Omega \rightarrow Y_{B}$ by $g(\omega)=\sum_{i \in N} J\left(c_{i}\right) E_{i}(\omega)$ for every $\omega \in \Omega$ such that $\sum_{i \in N}\left|J\left(c_{i}\right)\right|_{B} E_{i}(\omega)<\infty$, and let $g(\omega)=0$ otherwise. Then $g \in \mathfrak{B}\left(\lambda, Y_{B}\right)$ and $(4)$ holds.

THEOREM 6. Let $X$ be a Banach space, and let $J$ be a continuous embedding from $X$ into a sequentially complete locally convex space $Y$. Let $\lambda: \mathfrak{A} \rightarrow[0, \infty]$ be a non-negative measure. 
If $J$ is completely summing, then the space $\mathfrak{L}(\lambda ; J, X, Y)$ is complete.

Proof. It suffices to show that for every sequence $\left\langle s_{n}\right)_{n \in \mathbb{N}}$ in $\operatorname{sim}\left(\mathfrak{A}_{\lambda}, X\right)$ which is Cauchy in mean, $\left\langle J \circ s_{n}\right\rangle_{n \in N}$ converges in $\mathfrak{L}(\lambda ; J, X, Y)$. Let $\Pi(\lambda)$ be the collection of all families of finitely many pairwise disjoint sets $E$ from $\mathfrak{A}$ which satisfy $\lambda(E)<\infty$. The set $\Pi(\lambda)$ is directed by refinement.

Let $Z$ be a Banach space with norm $|\cdot|_{z}$. Given a vector measure $m: \mathfrak{A} \rightarrow$ $Z$ absolutely continuous with respect to the scalar measure $\lambda$, for each $\pi \in$ $\Pi(\lambda)$ define the $Z$-valued $\mathfrak{A}$-simple function $E_{\pi}(m)$ by

$$
E_{\pi}(m)(\omega)=\sum_{A \in \pi}(m(A) / \lambda(A)) A(\omega), \quad \omega \in \Omega
$$

with the understanding that $0 / 0=0$. It is clear that $\left\|E_{\pi}(m) \lambda\right\|_{Z}(\Omega) \leq$ $\|m\|_{Z}(\Omega)$, and if $m$ has finite variation, then $\left|E_{\pi}(m) \lambda\right|_{Z}(\Omega) \leq|m|_{Z}(\Omega)$.

If $f: \Omega \rightarrow Z$ is a Bochner $\lambda$-integrable function, then

$$
\lim _{\pi \in \Pi(\lambda)}\left|\mathbf{E}_{\pi}(f \lambda)-f\right|_{1}=0
$$

which can be proved as in the case when $\lambda$ is finite [2, Lemma III.2.1].

Let $\Gamma$ be a subset of $X$ which separates points of $X$. It follows from Theorem 3 that the sequence $\left\langle J_{\Gamma} \cdot s_{n}\right\rangle_{n \in N}$ is convergent in mean to a function $f \in \mathfrak{L}\left(\lambda ; X, C^{\Gamma}\right)$. Choose, according to Proposition 5 , a set $B \in \mathfrak{S}$ and a function $g \in \mathfrak{B}\left(\lambda, Y_{B}\right)$ such that (4) holds. Then

$$
J \circ \mathbf{E}_{\pi}(f \lambda)=\mathbf{E}_{\pi}(g \lambda), \quad \pi \in \Pi(\lambda) .
$$

For every $n=1,2, \ldots$, there exist a member $\pi_{n}$ of $\Pi(\lambda)$ such that

$$
\mathbf{E}_{\pi}\left(s_{n} \lambda\right)=s_{n}, \quad \pi \geq \pi_{n}, \quad \pi \in \Pi(\lambda) .
$$

The Bochner seminorm on the space $\mathfrak{B}\left(\lambda, Y_{B}\right)$ will be denoted by $|\cdot|_{1}$. Since $\lim _{\pi \in \Pi(\lambda)}\left|\mathbf{E}_{\pi}(g \lambda)-g\right|_{1}=0$, it is possible to choose a sequence of elements $\kappa_{n}$ from $\Pi(\lambda)$ such that $\kappa_{n} \geq \pi_{n}$ and

$$
\left|\mathbf{E}_{\kappa_{n}}(g \lambda)-g\right|_{1}<2^{-n}, \quad n=1,2, \ldots
$$

Let $f_{n}=\mathbf{E}_{\kappa_{n}}(f \lambda), n=1,2, \ldots$. Then it follows from (6) that $\left\|f-f_{n}\right\| \leq$ $2\left\|f-s_{n}\right\|, n=1,2, \ldots$. Since $\left\langle J_{\Gamma} \circ s_{n}\right\rangle_{n \in N}$ is convergent in mean to $f$ we may assume that $\sum_{n \in N}\left\|f_{n+1}-f_{n}\right\|<\infty$. It follows from (5) and (7) that $\sum_{n \in N}\left|J \circ f_{n+1}-J \circ f_{n}\right|_{1}<\infty$. 
Therefore the set $\Gamma$ of all $\omega \in \Omega$ for which $\sum_{n \in N}\left|J \circ f_{n+1}(\omega)-J \circ f_{n}(\omega)\right|_{B}<$ $\infty$ has full $\lambda$-measure. Define the function $h: \Omega \rightarrow Y$ by

$$
h(\omega)=\sum_{n \in N}\left(J \circ f_{n+1}(\omega)-J \circ f_{n}(\omega)\right)+J \circ f_{1}(\omega)
$$

for all $\omega \in \Gamma$ and let $h(\omega)$ be zero otherwise.

Then it follows from [4, Theorem 3] that $h \in \mathfrak{L}(\lambda ; J, X, Y)$ and that $h \lambda=$ $g \lambda$. The sequence $\left\langle J \circ s_{n}\right\rangle_{n \in N}$ is convergent in mean to $h$, and so the space $\mathfrak{L}(\lambda ; J, X, Y)$ is complete.

That the converse of Theorem 6 is not valid may be seen by taking any infinite dimensional Banach space $X$ and setting $Y=\mathbb{C}^{\Gamma}$ with $\Gamma=X^{\prime}$. The space $\mathfrak{L}\left(\lambda ; J_{\Gamma}, X, Y\right)$ is complete for any non-negative measure $\lambda$, but by the Dvoretsky-Rogers theorem, there always exists in $X$ an unconditionally summable sequence which is not absolutely summable, so the natural injection from $X$ into $Y$ is not completely summing.

The following proposition can be proved by the arguments used to show Theorem 4.7 in [8], and its proof is omitted.

Proposition 7. Let $X$ be a Banach space and let $J$ be a continuous embedding of $X$ into a locally convex Hausdorff space $Y$. If there exists a non-atomic, finite measure $\lambda$ such that the space $\mathfrak{L}(\lambda ; J, X, Y)$ is complete with respect to the topology of convergence in mean, then $J$ is absolutely summing.

The converse of Proposition 7 is not valid for arbitrary locally convex spaces $Y$. Let $Y$ be the space $X$ equipped with the weak topology. Then the identity map on $X$ is absolutely summing from $X$ into $Y$; nevertheless, the space $\mathfrak{L}(\mu ; X, Y)$ is not complete for the Lebesgue measure $\mu$, since $\mathfrak{L}(\mu ; X, Y)$ is identical to the space of all $X$-valued strongly measurable, $\mu$-Pettis integrable functions [4, Proposition 14], which is not complete [2, Theorem VIII.1.5].

In the case when $Y$ is a Banach space, an absolutely summing injection $J$ from $X$ into $Y$ is totally summing, so $\mathfrak{L}(\lambda ; J, X, Y)$ is complete. However, if $X$ is a non-separable and reflexive Banach space, then there exists no Banach space (and therefore no metric, or dual metric space) $Y$, for which there exists an absolutely summing embedding of $X$ into $Y$ [5, Corollary 7].

EXAMPLE 8. Let $\Omega$ be an open subset of $\mathbf{R}^{n}, n=1,2, \ldots$ Let $\mathfrak{D}(\Omega)$ be the linear space of all smooth functions on $\Omega$ with compact support, endowed with the usual inductive limit topology. Let $\mathfrak{D}(\Omega)^{\prime}$ be the dual of $\mathfrak{D}(\Omega)$, equipped with the strong dual topology. Then $\mathfrak{D}(\Omega)^{\prime}$ is conuclear with respect 
to the family of all bounded disked subsets of $\mathfrak{D}(\Omega)^{\prime}[7$, Theorem IV.1, Part II].

The space $C(\Omega)$ of all bounded continuous functions on $\Omega$ is endowed with the uniform norm. The Lebesgue measure on $\Omega$ is denoted by $\mu$. Define the linear map $J: C(\Omega) \rightarrow \mathfrak{D}(\Omega)^{\prime}$ by

$$
\langle J(f), \varphi\rangle=\int_{\Omega} f(\omega) \varphi(\omega) d \mu(\omega), \quad \varphi \in \mathfrak{D}(\Omega),
$$

for every $f \in \mathbf{C}(\Omega)$.

Then $J$ is a continuous linear injection. To see that it is totally summing, we observe that nuclear maps are absolutely summing, and from the definition of conuclear spaces, there exists a closed, bounded disked subset $B$ of $\mathfrak{D}(\Omega)^{\prime}$ for which $J(\mathbf{C}(\Omega)) \subseteq \mathfrak{D}(\Omega)_{B}^{\prime}$ and $J: \mathbf{C}(\Omega) \rightarrow \mathfrak{D}(\Omega)_{B}^{\prime}$ is nuclear.

It now follows from Theorem 6 that the space $\mathfrak{L}\left(\lambda ; J, C(\Omega), \mathfrak{D}(\Omega)^{\prime}\right)$ is complete with respect to the topology of convergence in mean, for every nonnegative measure $\lambda$.

\section{Acknowledgement}

The authors wish to thank Igor Kluvanek for pointing out ambiguities in an earlier version of this paper, and for suggesting improvements in its presentation.

\section{References}

[1] N. Bourbaki, Espaces vectorielles topologiques (Éléments de Mathématique, Livre V, Hermann, Paris, 1966).

[2] J. Diestel and J. J. Uhl, Jr., Vector measures (Math Surveys 15, Amer. Math. Soc., Providence, R. I., 1977).

[3] J. L. Kelley and T. P. Srinivasan, 'On the Bochner integral', Vector and operator valued measures and applications, pp. 165-174 (Academic Press, New York, 1973).

[4] S. Okada, 'Integration of vector valued functions', Measure theory and its applications, pp. 247-257 (Lecture Notes in Math., vol. 1033, Springer-Verlag, Berlin, Heidelberg, New York, Tokyo, 1983).

[5] S. Okada and Y. Okazaki, 'Injective absolutely summing operators', Math. Proc. Cambridge Philos. Soc., to appear.

[6] A. Pietsch, Nuclear locally convex spaces (Springer-Verlag, Berlin, Heidelberg, New York, 1972). 
[7] L. Schwartz, Radon measures in arbitrary topological spaces and cylindrical measures (Oxford University Press, London, 1973).

[8] G. E. F. Thomas, The Lebesgue-Nikodym theorem for vector-valued Radon measures, Mem. Amer. Math. Soc. 139 (1974).

Department of Mathematics

The University of Wollongong

Wollongong, N.S.W. 2500

Australia
School of Mathematical and Physical Sciences Murdoch University W.A. 6150

Australia 